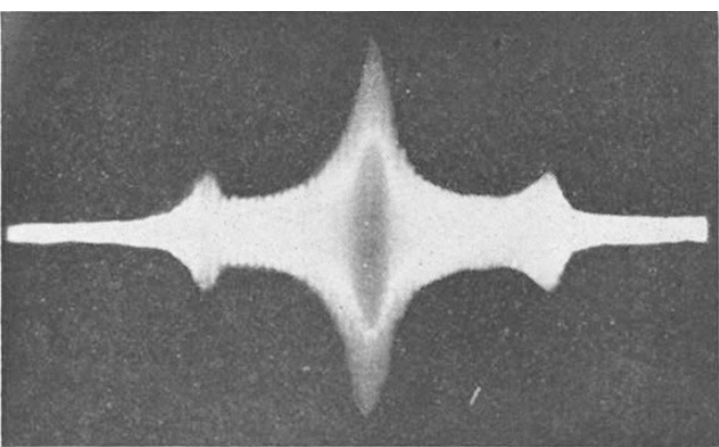

The first phenomenon is that of multiple resonance $e^{1,2}$ and is illustrated in the accompanying oscillogram for a circuit tuned to $6 \mathrm{kc} . / \mathrm{s}$., the resistive element of which was being varied at $50 \mathrm{c} / \mathrm{s}$. by means of a negative resistance. The abscissa represents the frequency of a driving E.M.F. and the ordinate the associated voltage (which varies in amplitude at $50 \mathrm{c} . / \mathrm{s}$.) across the main circuit capacitance; the average circuit resistance remained positive throughout the $50 \mathrm{c} . / \mathrm{s}$. variation. It can be shown that peak responses occur at frequencies $f_{0} \pm n f_{1}$, where $f_{0}=1 / 2 \pi \sqrt{\overline{L C}}=6 \times 10^{3} \mathrm{c.} / \mathrm{s}$., $f_{1}=50 \mathrm{c} / \mathrm{s}$. and $n=1,2,3 . \ldots$ The photograph shows only three such peaks, one below and one above $f_{0}$, but as many as six below and six above have been observed. The selectivity in the region of the central response increases with the amplitude of the resistance variation.

The second phenomenon is that of parametrically excited oscillations ${ }^{3,4}$ resulting when the effective circuit resistance varies at a frequency $f_{R} \approx 2 f_{0}$, and at the same time the resistance is allowed to become negative during part of its eyclic variation. Oscillations of substantially constant amplitude and purity of wave-form exactly half the frequency of the resistance variation $f_{R} / 2$ occur over a range of frequencies near $f_{0}$. The range is limited by sharp cut-off of the self-oscillation, and this suggests the use of the device as a filter, particularly since the band-width is readily controlled.

The theoretical basis of these phenomena lies in Hill's equation ${ }^{5}$ :

$$
\ddot{y}+[a-2 q \psi(2 z)] y=F(z),
$$

where $a$ and $q$ are constants, and $\psi(2 z)$ and $F(z)$ are periodic functions of time. Good quantitative agreement has been obtained between experiment and, on one hand, multiple resonance calculations from the particular integral solution, and on the other, calculation of the self-oscillation frequency and band-width obtained from the solution in the unstable $a-q$ region of the complementary function. Full particulars will be given in a later publication.

We are indebted to E. H. Ballantyne and L. S. Dektereff for assistance with the experimental work. P. BURA D. M. Томвs

Electrical Engineering Department, Imperial College, London, S.W.7.

${ }^{2}$ Gorelik, G., and Hintz, G., Z. Hochfreq., 38, 222 (1931)

2 Gluckman, H. A., Proc. Inst. Rad. Eng., 37, No. 5, 500 .

${ }^{3}$ Winter-Günther, H., Z. Hochfreq., 37, 172.

- Mandlelstam and Papalexi, J. Tech. Phys., U.S.S.R., 3, 1141 (1933) 4, No. 1, 5 (1934).

Ince, E. L., Mon. Not. Roy. Astro. Soc., 75, 436 (1915).

\section{An Improved Type of Wetted-Wall Column}

Preliminary investigations, carried out in this Department, have shown that the efficiency of a wetted-wall column may be improved by subjecting the liquid and vapour phases to a rapidly oscillating pressure. This induces increased turbulence in the refluxing liquid, resulting in a larger surface area for mass transfer.

A wetted-wall column was attached, via the condenser, to a cylinder closed by a reciprocating piston. Pressure changes of the order of 15 per cent at a frequency of 20 cycles per second have resulted in an increased efficiency of approximately 50 per cent, expressed in terms of theoretical plates.

The efficiency of a column 'packed' with a gauze spiral may also be increased by this device, but to a lesser extent, dependent upon the packing density.

Dept. of Chemical Engineering, JOHN D. THORNTON

University, Birmingham 15. May 17.

\section{Occurrence of a Network in the Excrement from the Larva of the Clothes Moth}

As a detail in an investigation of the degradation products from enzymatic digestion of wool, excrement from the larva of the clothes moth (Tineola biselliella Hummel) have been subjected tö examination in the electron microscope.

The pellets were ground in an agate mortar and suspended in water. A droplet of the suspension was placed on the collodion film, dried and shadowcast with gold-manganin. The examination was carried out with an $R C A$ microseope, type $E M U$.

Among various stages of disintegrated wool particles very regular networks were also observed, often covering areas with diameters up to more than $50 \mathrm{u}$. Three different main types could be recognized. The first one (Fig. 1) is the regular 'fishing-net' pattern, constituted by threads or fibres running in three main directions, thus forming symmetrical meshes with a width of about $0 \cdot 2 \mu$. These fibres are themselves bundles of fibrils, each of which has a thickness of 50-60 A. At the junctions it can be seen how the fibrils from different threads are plaited. The fibrils can also be observed in the places where the fibres are split up, for example, near the borders of the network. The regularity of the pattern is well preserved even near the border. 'The second type is shown in Fig. 2. Basically, it consists of the same network, which can be recognized on the photograph ; but here the meshes are filled up with some substance so that a continuous background membrane is formed. The white formations are bigger, extraneous particles lying underneath the membrane. In the third type, shown in Fig. 3, the fibrils seem to be arbitrarily arranged and the regular pattern has been destroyed, at least partly. Parts of the network, forming stages between the first and third types, have also been observed.

It may be worth mentioning that these networks were very searce if the specimen was prepared immediately after suspending the ground pellets in water. If, however, the same suspension was allowed to stand for about two days at room temperature, the networks were both bigger and more abundant. It seems probable that the network originates from the intestines of the moth larvæ, as we have also 